\title{
The implementation of mental health information systems in developing countries: Challenges and opportunities
}

\author{
DAVID M. NDETEI and RACHEL JENKINS
}

\begin{abstract}
The aims are to examine the challenges and opportunities in the implementation of mental health information systems (MHIS) in developing countries as suggested by the World Health Organization (WHO) and explored by Gulbinat et al. (2008). Special recommendations for developing countries are: 1) MHIS should be linked to the general medical information system; 2) there is need for adoption, adaptation and validation of preferably self-administered instruments that are appropriate for different levels within the health care system; 3 ) developing countries must adopt innovative and "unconventional" approaches through utilization of community members, traditional doctors/healers and mid-cadre health workers, in addition to general doctors, for the delivery of mental health services.
\end{abstract}

Declaration of Interest: None.

\section{THE PURPOSES, FUNCTIONS AND COMMON FRAMEWORK OF MHIS}

The comprehensive World Health Organisation (WHO) definition of Mental Health Information Systems (MHIS) (World Health Organization, 2005) explored in Gulbinat et al. (2008) emphasized the need for effective patient management, policy and service development, monitoring, evaluation, research prioritization and implementation. These are however dependent on local contextual factors which vary from community to community within the same country and from one country to another.

Nonetheless it is possible to construct a common framework of useful information at individual, local and national levels. At individual level, these data include socio-demographic characteristics, and clinical information such as diagnosis, severity, disability, chronicity, family history, laboratory tests, treatment outcome (expected or achieved) and service utilization. The individual data are amalgamated to generate data for the primary, secondary and tertiary care and national levels.

The core building block of MHIS is the individual data

Address for correspondence: Professor D. Ndetei, University of Nairobi, Africa Mental Health Foundation, Ralph Bunch Road P.O. Box, 48423, 00100 GPO, Nairobi (Kenya).

Fax: +254-(2)-271.6315

E-mail: dmndetei@uonbi.ac.ke which must be valid, reliable, and, reasonably comprehensive by adopting the multiaxial bio-psychosocial model for onset, course and recovery. Validity, reliability and comprehensiveness demand a common language beginning at the individual level.

Clinical data can be complemented with epidemiological survey data on prevalence, severity, disability and chronicity of mental disorders in community and special groups. Hence the need for common terminologies, agreed definitions and approved categorical and/or dimensional classifications.

\section{The Challenges}

The challenges are many. Firstly is the collection and safe storage of data, confidentiality, accessibility and retrievability. Secondly, data collection is time-consuming and requires human resources, training and availability of storage technology, i.e. electronic or paper and pencil. Electronic systems are often expensive to set up, maintain and require electricity which is sparsely available. Health facilities at the primary care level, and often at the district level in low income countries currently rely on paper and pencil systems of data collection. Thirdly, there is prioritization which relegates data in low income countries to only one category for mental disorders, which is useless for planning purposes, as the different mental disorders have differing prevalence rates, severity, disability and require different treatment models.

Further classification of mental disorders has always 
been more contentious than classification of physical illness. Also attaching diagnostic labels may be stigmatising and socially harmful. More difficult to standardize are the unique socio-economic, cultural and environmental contexts which vary widely within and without countries.

\section{INTERNATIONAL AND OTHER CLASSIFICATIONS AND THEIR CHALLENGES}

There are two main international classification systems in current use: The $10^{\text {th }}$ revision of the International Statistical Classification of Diseases and Related Health Problems (ICD-10; a WHO publication) and the fourth edition of the American Psychiatric Association's Diagnostic and Statistical Manual of Mental Disorders (DSM-IV). WHO has also produced instruments for standardizing classification of functioning, health intervention and descriptions for clinical and diagnostic purposes in mental and behavioural disorders and also for neurology. In a third one, the WHO focuses on standardization for special areas, namely primary care at general and family practice levels and external causes of injury and disability.

In addition to the above, the United Nations (UN) has classifications which provide important information with general and specific relevance to health in general and mental health in particular. These include classification of all economic activities, central products, status of employment, occupations, education, activities for time-use statistics (working in "formal" sector employment, household production activities, socializing and community participation, unpaid caregiving services to household members) and functions of the government.

Several other classifications that are relevant to mental health are also widely available and in use. These include the International Classification for Nursing Practice by the International Council of Nurses and the systematized nomenclature of medicine by the International Health Terminology Development Organisation.

There are other classifications used in specific regions. Examples include the Chinese Classification of Mental Disorders, intentionally similar to DSM and ICD but with some particular minor variations that include about 40 culturally-related diagnoses; the Cuban Glossary of Psychiatry; the Latin American Guide to Psychiatry and the French Classification of Child and Adolescent Mental Disorders.

\section{The challenges}

i) Compatibility of Classifications: There are challenges of compatibility between two successive versions of the same classification; vertical and horizontal compatibility between the different classifications. For example, how does the current version of the ICD-10 (in existence since 1989) compare with ICD-9 (came into existence in 1975) and sequentially in respective editions going back to the first ICD1 edition of 1900 (108 years ago)? How will ICD-10 compare with ICD-11 expected in 2011? If there is a relative vertical incompatibility in diagnoses by either narrowing or broadening the diagnostic criteria for a given condition, then this is bound to reflect on clinical practice, epidemiological patterns and policies.

Horizontal compatibility seeks to measure how compatible or incompatible different classifications (e.g. between ICD-10 \& DSM-IV) are on the same phenomenon, whether for routine clinical practice or research diagnoses. The other facet of horizontal compatibility concerns how different classifications complement each other in providing a holistic approach to the mental health aspects and measurement of a given individual, community or country. Thus, for a given psychiatric diagnosis, the level of disability, functioning and intervention are inter-linked and all have an effect on the outcome.

ii) Time Lag between Revision and Use of Revised Classifications: There is a time lag in the implementation of the successive versions of the same classification. Although ICD-10 was released by the WHO in 1989 , by 2005 ( 16 years later) and 6 years to the expected release of ICD-11, 14\% of all the countries in the world reporting ICD in information gathering systems, still used ICD-9. This delay is occasioned by the technicalities and major costs involved in shifting to the new coding systems (training of personnel, and upgrading of electronic or paper-based systems) and the intended use (mortality or morbidity and related issues or reimbursement and insurance) of the ICD classification. Even in resource-rich countries such as Australia, many datasets used ICD9 as late as 2001 and by the late 1990s were using both ICD-9 and ICD-10. In the UK, use of ICD-10 for the National Health System (NHS-2007) is mandatory while in the USA, since 1999 ICD-10 was used for mortality reporting but not for morbidity (Gulbinat et al., 2008). 
The consequences of delayed implementation of different systems at different time points in different countries are that there will be problems in comparability of data from different countries at any particular time and over particular periods of time.

iii) Differing levels of complexity of classifications for use at different levels of the health services: Primary level care in resource-rich countries is usually undertaken by general and family doctors (who have usually received specialist training in family medicine after their basic medical training, are supported by trained nurses and administrators, and who have demonstrated ability to make multiaxial assessments) (Gulbinat et al., 2008). In low income countries, clinical services at primary care (dispensary and health centre) and district levels are usually delivered by nurses, mid-cadre personnel and relatively untrained volunteers without training and orientation in mental health and who are therefore deficient in recognizing mental health disorders, let alone making diagnoses for effective management information. The situation is particularly difficult for mental health workers who are extremely sparsely distributed in low income countries. Taking Kenya as an example of a low income country, with a population of around 35 million, there are 72 psychiatrists of whom less than half are in the public service while most are based in the capital city. There is at least one psychiatrist for six out of the seven provinces outside Nairobi, but only 7 out of 140 districts have a psychiatrist. There are about 250 psychiatric nurses in Kenya, and half of these are deployed into non-psychiatric posts to alleviate staffing shortages elsewhere (Gulbinat et al., 2008).

Despite the weight given to physical and life-threatening conditions, significant mental health disorders co-exist with and complicate physical conditions (Ndetei et al., in press), legitimizing the need for paying attention to mental disorders as well. There is therefore a need for in-service training on mental disorders for staff manning general facilities. Traditional healers who are widely available at grass-root level are able to accurately recognize simple mental disorders and these skills can be improved with training (Ndetei et al., 2008).

iv) Multiaxial classification and implications for MHIS: As has already been argued, multi-axial classification has merit. In reality however, most mental health information systems usually use a uni-axial approach. Furthermore, comparability between countries in, for example, contextual factors and what is considered normal social and environmental contexts and even quality of life, would vary in content and perception. This makes comparability amongst all systems impossible. People who live on less than one dollar a day (whose preoccupation in life is when and where to get their next meal), will have very different perceptions of acceptable social and environmental contexts and quality of life compared with those whose preoccupation is to cut down on over-eating and are concerned with when or where to take their next vacation. This means that multi-axial classification is only meaningful if it is contextualized. It therefore does not lend itself to easy comparability across different contexts, even within resource-rich countries as well as within the resource-poor countries.

\section{The Opportunities with Regard to Classification}

The first one is to empower nurses, clinical officers and community health workers (at primary health care level and at district level) to recognize mental health disorders, and apply the simple classifications described above, and then accord them the opportunity of recording these diagnoses on the pencil and paper tally sheets in use in the clinics. Secondly, there is scope to encourage the systematic documentation of other important aspects, such as functioning in the community, productivity and recognition of and dealing with disabilities related to mental disorders. Thirdly, efforts should be made to educate the lay community traditional healers to recognize people with simple psychiatric disorders within their families and clientele and assist them to improve their record-keeping skills. Fourthly, there should be emphasis on sensitizing general nurses, clinical officers and doctors on mental health issues during their basic and post-basic training and continuing education. In this sensitization, an orientation to the systems of classification of mental disorders should be included.

\section{AVAILABILITY, RETRIEVABILITY, RELIABILITY AND VALIDITY OF INFORMATION: CHALLENGES AND OPPORTUNITIES}

Availability and retrievability present special difficulties not only in mental health but also in other medical 
conditions where records are poorly kept as hard copies at the primary health care levels where there is no electricity. It is at this level where data that generally should reflect the country profile are generated and it is even more complicated if different institutions use different systems of classification. Training on record-keeping is an opportunity that could be linked to training on recognition of mental disorders.

One of the practicable ways of increasing reliability is to use survey, research and psychiatric assessment instruments that have a proven test-retest agreement. They should be simple to use and should be adapted and then their psychometric properties tested in different contexts. They should also be designed and couched in a language that is easily understood, preferably be selfadministered or simply read out to the respondent by a trained lay person. This approach provides the greatest window of opportunity for resource-poor countries where lay people will, out of necessity, play a significant role in recognizing and classifying mental health disorders.

Validity is affected by several limitations: the level of training of the clinician; the common structured language for given disorders and the specificity levels of the actual health information systems and instruments; their applicability across different socio-cultural contexts, whether paper and pencil or sophisticated software; the clinician's workload; and, determination of what constitutes first-contact and drop-out. None of the above limitations has been brought under control and/or supported by evidence even in the best resourced centres. This situation is likely to be worse in poorly-resourced countries. There is therefore need to find what best works for a given situation (given the peculiar socio-economic realities) and how what works can be improved. This is the challenge that is faced by developing countries and provides opportunities for research for evidence for the most realistic practices.

\section{OTHER CHALLENGES AND OPPORTUNITIES}

\section{i) Linking Mental Health to Other Information Systems}

Mental and physical disorders are co-morbid. In general medical settings, mental disorders tend to be neglected or are not recognized whereas in psychiatric settings, the reverse happens. Linking mental health to other information systems and vice-versa is desirable for the clinical and holistic approach to management.
Such linkages will allow mental health to benefit from the better funded and easily acceptable information systems that are in place for physical conditions. However, in order to be more meaningful, the linkages should not just be separate systems within the same institution but one system that is also able to link data to a particular individual.

\section{ii) The Electronic Health Record (EHR) For Mental Health}

The potential of the EHR is not in doubt, despite the increased challenges of confidentiality and accessibility by unauthorized persons. The EHR is still expensive in terms of infrastructure and the need for highly trained people and although increasingly feasible, it is not yet widely available. This is likely to take much longer to realize in developing countries where electricity is available mainly in urban and peri-urban areas where minority of the population live. However there is a window of opportunity in that the use of the EHR can be initiated on a small scale experimental basis at the tertiary levels since training also takes place here. The experiences can be passed on to the trainees and rolled out as resources become more available.

\section{RECOMMENDATIONS}

From the perspective of resource-poor countries, the recommendations arising out of the Gulbinat et al. (2008) paper are welcome but only if three of these are given the prominence they deserve because the feasibility of implementing the others depends entirely on these special recommendations.

1. Adaptation, validation and standardization of screening and diagnostic psychometric tests in the various socio-cultural contexts in developing countries - this will also create the basis for comparability of data across countries. This is an area that is vital, and coordination between researchers will help to avoid duplication of efforts. The WHO should provide leadership in this area.

2. The above will go hand in hand with sensitization and training of personnel. This sensitization should start in all health training curricula, and all personnel will need to be familiar with essential record keeping.

3. Mental health information systems in all countries and particularly, in developing and resource-poor coun-

Epidemiologia e Psichiatria Sociale, 18, 1, 2009 
tries, make efforts to ensure the inclusion of mental health in general MHIS, as this will be funded from general sources.

Apart from the above, it is particularly recommended for the case of developing countries to include trials and evaluation of educating basic health workers, volunteer health workers, and even the self-help community level support groups, and traditional health practitioners to recognize organic disorders, psychotic conditions, substance abuse, depressive and anxiety disorders. They can be trained not only to record these but also on how to recognize the disabilities, dysfunctions and effects that may arise in the lives of their relatives and community members with these conditions in a way that clinicians may not be able to discern or even perceive. Ndetei et al. (2008) and Chatterjee al. (in press) have provided ample evidence for this.

Acknowledgements. Patricia Wekulo and Grace Mutevu of the Africa Mental Health Foundation provided editorial and secretarial assistance respectively, and David Kiima, Ministry of Health, Kenya provided moral support.

\section{REFERENCES}

Chatterjee S., Pillai A., Jain S., Cohen A. \& Patel V. (in press). Outcomes of people with psychotic disorders in a community based rehabilitation program in rural India. British Journal of Psychiatry.

Gulbinat W., Amaddeo F., Ito H., Medina-Mora E., Mubbashar M., Ndetei D. \& Plovnick R. (2008). Statistics and Information Systems. Background Paper from the Statistics and Information Systems Conference Expert Group (CEG) for the Conference on Public Health Aspects of Diagnosis and Classification. Version 31 May 2008.

Ndetei D.M., Khasakhala L.I., Kingori J., Oginga A. \& Raja S. (2008). The complementary role of traditional and faith healers and potential liaisons with western-style mental health services in Kenya Retrieved December 1, 2008, from http://www.utsouthwestern.edu/psychiatryandfreedom

Ndetei D.M., Khasakhala L.I., Kuria M.W., Mutiso M.N., OngechaOwuor F.A. \& Kokonya D.A. (in press). The prevalence of mental disorders in adults in different level general medical facilities in Kenya: A cross-sectional study. Annals of General Psychiatry.

World Health Organization (2005). Mental Health Information Systems. WHO: Geneva. 